\title{
Metrizable compacta in the space of continuous functions with the topology of pointwise convergence
}

\author{
V.V. Mykhaylyuk ${ }^{1}$ \\ Chernivtsi National University, Department of Mathematical Analysis, \\ Kotsjubyns'koho 2, Chernivtsi 58012, Ukraine \\ e-mail:mathan@ukr.net
}

\begin{abstract}
We prove that every point-finite family of nonempty functionally open sets in a topological space $X$ has the cardinality at most an infinite cardinal $\kappa$ if and only if $w(X) \leq \kappa$ for every Valdivia compact space $Y \subseteq C_{p}(X)$. Correspondingly a Valdivia compact space $Y$ has the weight at most an infinite cardinal $\kappa$ if and only if every point-finite family of nonempty open sets in $C_{p}(Y)$ has the cardinality at most $\kappa$, that is $p\left(C_{p}(Y)\right) \leq \kappa$. Besides, it was proved that $w(Y)=p\left(C_{p}(Y)\right)$ for every linearly ordered compact $Y$. In particular, a Valdivia compact space or linearly ordered compact space $Y$ is metrizable if and only if $p\left(C_{p}(Y)\right)=\aleph_{0}$. This gives answer to a question of O. Okunev and V. Tkachuk.
\end{abstract}

\section{Introduction}

During the study of the Baire classification of separately continuous functions $f: X \times Y \rightarrow \mathbb{R}$, when $Y$ satisfies compactness type conditions, it was established that spaces $X$ which satisfy a condition of a countable chain type, play an important role (see $[1,2,3])$. The Baire classification of these mappings was obtained replacing of $Y$ with its continuous metrizable image in the space $C_{p}(X)$ of all continuous functions on $X$ with the pointwise convergence topology.

This shows that it is natural to try to find necessary and sufficient conditions on a topological space $X$ for metrizability of any compact subspace of $C_{p}(X)$

$\overline{1}$ Key words and phrases: Valdivia compact, metrizable compact, separately continuous function, point-finite cellularity, linearly ordered compact.

2000 Mathematics Subject Classification: Primary 54E45, 54C35; Secondary 54A25, $54 \mathrm{D} 30$

Preprint submitted to Acta Mathematica Hungarica

2 May 2018 
or any compact subspace of $C_{p}(X)$ which belongs to a given class such as Eberlein compact spaces, Corson compact spaces or Valdivia compact spaces.

A compact space $X$ which is homeomorphic to a weakly compact subset of a Banach space is called an Eberlein compact space. A compact space $X$ is called a Corson compact space if it is homeomorphic to a compact space $Z \subseteq \mathbb{R}^{T}$ such that $|\operatorname{supp} z| \leq \aleph_{0}$ for any $z \in Z$. By $\operatorname{supp} z$ we denote the support $\{t \in T: z(t) \neq 0\}$ of a function $z: T \rightarrow \mathbb{R}$. A compact space $X$ is called a Valdivia compact space if it is homeomorphic to a compact space $Z \subseteq \mathbb{R}^{T}$ such that the set $\left\{z \in Z:|\operatorname{supp} z| \leq \aleph_{0}\right\}$ is dense in $Z$.

Analogous questions were investigated in $[4,5,6,7]$, where connections between cardinal properties of a completely regular space $X$ and the space $C_{p}(X)$ were studied. The point-finite cellularity $p(X)=\sup \{|\tau|: \tau$ is a point-finite family of nonempty open sets in $X\}$ of a topological space $X$, which was introduced in [4], is important in these papers. In particular, the following questions were posed in $[7$, questions $5.2,5.4]$ :

Question 1.1 Let $X$ be a Souslin continuum, i.e. a non-separable linearly ordered perfectly normal compact space. It is true that $p\left(C_{p}(X)\right)=\aleph_{0}$ ?

Question 1.2 Let $X$ be a Corson compact space such that $p\left(C_{p}(X)\right)=\aleph_{0}$. Must $X$ be metrizable?

Question 1.2 was answered in [8]. It is proved in [8] that the weight of a Corson compact space $X$ is equal to the point-finite cellularity of $C_{p}(X)$.

In this paper we study necessary and sufficient conditions on a topological space $X$ for metrizability of any Valdivia compact space $Y \subseteq C_{p}(X)$. Using a technique of a dependence of mappings upon a certain number of coordinates we prove that any point-finite family of functionally open sets in a topological space $X$ has the cardinality at most an infinite cardinal $\kappa$ if and only if each Valdivia compact space $Y \subseteq C_{p}(X)$ has the topological weight at most $\kappa$. In particular, $p(X)=\aleph_{0}$ for a completely regular space $X$ if and only if each Valdivia compact space $Y \subseteq C_{p}(X)$ is metrizable. Passing to a dual problem on study of connections between properties of a compact space $Y$ and the space $C_{p}(Y)$, we obtain that a Valdivia compact space $Y$ has the weight at most an infinite cardinal $\kappa$ if and only if $p\left(C_{p}(Y)\right) \leq \kappa$. It implies immediately the following fact: a Valdivia compact space $Y$ is metrizable if and only if $p\left(C_{p}(Y)\right)=\aleph_{0}$. Besides, it was proved that $w(Y)=p\left(C_{p}(Y)\right)$ for every linearly ordered compact space $Y$ (this yields a negative answer to Question 5.2 from $[7])$. 


\section{A dependence of mappings upon a certain number of coordinates}

In this section we prove a theorem which will be a technical tool in the proof of the main result and based on a notion of a dependence of mappings upon a certain number of coordinates.

Let $\kappa$ be an infinite cardinal number, and let $Z, T$ be sets, $Y \subseteq \mathbb{R}^{T}$ and $f: Y \rightarrow Z$. We say that $f$ is concentrated on a set $S \subseteq T$ if for any $y^{\prime}, y^{\prime \prime} \in Y$ the condition $\left.y^{\prime}\right|_{S}=\left.y^{\prime \prime}\right|_{S}$ implies $f\left(y^{\prime}\right)=f\left(y^{\prime \prime}\right)$. Besides, if $|S| \leq \kappa$ then $f$ depends upon $\kappa$ coordinates.

Let $X$ be a set and $g: X \times Y \rightarrow Z$. Then $g$ is concentrated on a set $S \subseteq T$ in the second variable if $g\left(x, y^{\prime}\right)=g\left(x, y^{\prime \prime}\right)$ for any $x \in X$ and $y^{\prime}, y^{\prime \prime} \in Y$ with $\left.y^{\prime}\right|_{S}=\left.y^{\prime \prime}\right|_{S}$; and $g$ depends upon $\kappa$ coordinates in the second variable if $|S| \leq \kappa$ for some $S$.

A subset $A$ of a topological space $X$ is called functionally open if there exists a continuous function $f: X \rightarrow[0,1]$ such that $A=f^{-1}((0,1])$.

The following result was obtained in [9] for $\kappa=\aleph_{0}$.

Theorem 2.1 Let $X$ be a topological space in which every point-finite family of nonempty functionally open sets has the cardinality at most an infinite cardinal number $\kappa$, let $Y \subseteq \mathbb{R}^{T}$ be a compact space, $B=\{y \in Y:|\operatorname{supp} y| \leq \kappa\}$ and $Y=\bar{B}$. Then every separately continuous function $f: X \times Y \rightarrow \mathbb{R}$ depends upon $\kappa$ coordinates in the second variable.

Proof. First we prove that for every $\varepsilon>0$ there exists a set $R_{\varepsilon} \subseteq T$ such that $\left|R_{\varepsilon}\right| \leq \kappa$ and for any $b^{\prime}, b^{\prime \prime} \in B$ the condition $\left.b^{\prime}\right|_{R_{\varepsilon}}=\left.b^{\prime \prime}\right|_{R_{\varepsilon}}$ implies $\left|f\left(x, b^{\prime}\right)-f\left(x, b^{\prime \prime}\right)\right| \leq \varepsilon$ for every $x \in X$.

Suppose, contrary to our claim, that there exists an $\varepsilon>0$ such that for every set $S \subseteq T$ with $|S| \leq \kappa$ there exist an $x \in X$ and $b^{\prime}, b^{\prime \prime} \in B$ such that $\left.b^{\prime}\right|_{S}=\left.b^{\prime \prime}\right|_{S}$ and $\left|f\left(x, b^{\prime}\right)-f\left(x, b^{\prime \prime}\right)\right|>\varepsilon$.

Denote by $T\left(\kappa^{+}\right)$the first ordinal of cardinality $\kappa^{+}$. Using the transfinite induction we construct a system $\left\{S_{\alpha}: \alpha<T\left(\kappa^{+}\right)\right\}$of sets $S_{\alpha} \subseteq T$ and sets $\left\{b_{\alpha}: \alpha<T\left(\kappa^{+}\right)\right\},\left\{c_{\alpha}: \alpha<T\left(\kappa^{+}\right)\right\}$and $\left\{x_{\alpha}: \alpha<T\left(\kappa^{+}\right)\right\}$of points $b_{\alpha}, c_{\alpha} \in B$ and $x_{\alpha} \in X$ such that

(a) $\left|S_{\alpha}\right| \leq \kappa$ for any $\alpha<T\left(\kappa^{+}\right)$;

(b) $\left.b_{\alpha}\right|_{S_{\alpha}}=\left.c_{\alpha}\right|_{S_{\alpha}}$ for any $\alpha<T\left(\kappa^{+}\right)$;

(c) $S_{\alpha} \subseteq S_{\beta}$ for any $\alpha<\beta<T\left(\kappa^{+}\right)$; 
(d) $\operatorname{supp} b_{\alpha} \subseteq S_{\alpha+1}$, supp $c_{\alpha} \subseteq S_{\alpha+1}$ for any $\alpha<T\left(\kappa^{+}\right)$;

(e) $\left|f\left(x_{\alpha}, b_{\alpha}\right)-f\left(x_{\alpha}, c_{\alpha}\right)\right|>\varepsilon$ for any $\alpha<T\left(\kappa^{+}\right)$.

Fix a set $S_{1} \subseteq T$ with $\left|S_{1}\right| \leq \kappa$. By the assumption, there exist points $x_{1} \in X$ and $b_{1}, c_{1} \in B$ such that $\left.b_{1}\right|_{S_{1}}=\left.c_{1}\right|_{S_{1}}$ and $\left|f\left(x_{1}, b_{1}\right)-f\left(x_{1}, c_{1}\right)\right|>\varepsilon$. Put $S_{2}=S_{1} \cup \operatorname{supp} b_{1} \cup \operatorname{supp} c_{1}$. Clearly $\left|S_{2}\right| \leq \kappa$. Choose points $x_{2} \in X$ and $b_{2}, c_{2} \in B$ such that $\left.b_{2}\right|_{S_{2}}=\left.c_{2}\right|_{S_{2}}$ and $\left|f\left(x_{2}, b_{2}\right)-f\left(x_{2}, c_{2}\right)\right|>\varepsilon$.

Suppose that for some $\beta<T\left(\kappa^{+}\right)$the sets $\left\{S_{\alpha}: \alpha<\beta\right\},\left\{b_{\alpha}: \alpha<\beta\right\},\left\{c_{\alpha}\right.$ : $\alpha<\beta\}$ and $\left\{x_{\alpha}: \alpha<\beta\right\}$ are constructed. Put $S_{\beta}=\bigcup_{\alpha<\beta}\left(S_{\alpha} \cup \operatorname{supp} b_{\alpha} \cup \operatorname{supp} c_{\alpha}\right)$. Since for $\alpha<\beta$ all the sets $S_{\alpha}, \operatorname{supp} b_{\alpha}$ and $\operatorname{supp} c_{\alpha}$ have cardinality at most $\kappa$, we have the inequality $\left|S_{\beta}\right| \leq \kappa$. By the assumption, there exist points $x_{\beta}$ and $b_{\beta}, c_{\beta} \in B$ such that $\left.b_{\beta}\right|_{S_{\beta}}=\left.c_{\beta}\right|_{S_{\beta}}$ and $\left|f\left(x_{\beta}, b_{\beta}\right)-f\left(x_{\beta}, c_{\beta}\right)\right|>\varepsilon$.

The continuity of $f$ in variable $x$ and condition (e) imply that for every $\alpha<$ $T\left(\kappa^{+}\right)$there exists an functionally open neighborhood $U_{\alpha}$ of $x_{\alpha}$ in $X$ such that $\left|f\left(x, b_{\alpha}\right)-f\left(x, c_{\alpha}\right)\right|>\varepsilon$ for any $x \in U_{\alpha}$. According to the theorem condition, the family $\left(U_{\alpha}: \alpha<\omega\right)$ is not point-finite. Thus there exist a point $x_{0} \in X$ and a strictly increasing sequence $\left(\alpha_{n}\right)_{n=1}^{\infty}$ of ordinals $\alpha_{n}<T\left(\kappa^{+}\right)$such that $\left|f\left(x_{0}, b_{\alpha_{n}}\right)-f\left(x_{0}, c_{\alpha_{n}}\right)\right|>\varepsilon$ for any $n \in \mathbb{N}$.

Put $T_{n}=S_{\alpha_{n}}, v_{n}=b_{\alpha_{n}}$ and $w_{n}=c_{\alpha_{n}}$ by $n \in \mathbb{N}$. Using the compactness of $Y$ and the continuity of $f^{x_{0}}: Y \rightarrow \mathbb{R}, f^{x_{0}}(y)=f\left(x_{0}, y\right)$, choose a finite set $T_{0} \subseteq T$ such that $\left|f\left(x_{0}, y^{\prime}\right)-f\left(x_{0}, y^{\prime \prime}\right)\right|<\varepsilon$ for any $y^{\prime}, y^{\prime \prime} \in Y$ with $\left.y^{\prime}\right|_{T_{0}}=\left.y^{\prime \prime}\right|_{T_{0}}$. It follows from $\left|f\left(x_{0}, v_{n}\right)-f\left(x_{0}, w_{n}\right)\right|>\varepsilon$ that $\left.v_{n}\right|_{T_{0}} \neq\left. w_{n}\right|_{T_{0}}$. But $\left.v_{n}\right|_{T_{n}}=\left.w_{n}\right|_{T_{n}}$ according to $(b)$ and $\left.v_{n}\right|_{T \backslash T_{n+1}}=\left.w_{n}\right|_{T \backslash T_{n+1}}$ according to $(c)$ and $(d)$. Thus $T_{0} \cap\left(T_{n+1} \backslash T_{n}\right) \neq \varnothing$ for any $n \in \mathbb{N}$. Since the sequence $\left(T_{n}\right)_{n=1}^{\infty}$ is increasing, $T_{0}$ is infinite, which is impossible. Hence the existence of $R_{\varepsilon}$ is proved.

Put $R_{0}=\bigcup_{n=1}^{\infty} R_{\frac{1}{n}}$. Clearly $f\left(x, b^{\prime}\right)=f\left(x, b^{\prime \prime}\right)$ for any $x \in X, b^{\prime}, b^{\prime \prime} \in B$ with $\left.b^{\prime}\right|_{R_{0}}=\left.b^{\prime \prime}\right|_{R_{0}}$. Fix points $x \in X, y^{\prime}, y^{\prime \prime} \in Y$ such that $\left.y^{\prime}\right|_{R_{0}}=\left.y^{\prime \prime}\right|_{R_{0}}$. Since a function $f^{x}: Y \rightarrow \mathbb{R}, f^{x}(y)=f(x, y)$ is continuous on the compact space $Y \subseteq \mathbb{R}^{T}$, there exists an at most countable set $T_{0} \subseteq T$ such that $f\left(x, y_{1}\right)=$ $f\left(x, y_{2}\right)$ for any $y_{1}, y_{2} \in Y$ with $\left.y_{1}\right|_{T_{0}}=\left.y_{2}\right|_{T_{0}}$. Consider a continuous mapping $\varphi: Y \rightarrow \mathbb{R}^{T_{0} \cup R_{0}}, \varphi(y)=\left.y\right|_{T_{0} \cup R_{0}}$. Since $Y=\bar{B}$, we have the inequality $\varphi(Y)=\overline{\varphi(B)}$. Note that $B$ is an $\kappa$-compact set, that is, the closure $\bar{A}$ in $B$ of any set $A \subseteq B$ with $|A| \leq \kappa$ is a compact set. Therefore $\varphi(B)$ is an $\kappa$-compact set. It follows from $\left|T_{0} \cup R_{0}\right| \leq \kappa$ that $w(\varphi(Y)) \leq \kappa$. The density of $\varphi(B)$ in $\varphi(Y)$ implies the existence of a dense in $\varphi(Y)$ set $C \subseteq \varphi(B)$ such that $|C| \leq \kappa$. Then $\varphi(Y) \subseteq \bar{C} \subseteq \varphi(B)$. Thus $\varphi(Y)=\varphi(B)$. Therefore there exist points $b^{\prime}, b^{\prime \prime} \in B$ such that $\left.b^{\prime}\right|_{T_{0} \cup R_{0}}=\left.y^{\prime}\right|_{T_{0} \cup R_{0}}$ and $\left.b^{\prime \prime}\right|_{T_{0} \cup R_{0}}=\left.y^{\prime \prime}\right|_{T_{0} \cup R_{0}}$. Since $\left.b^{\prime}\right|_{T_{0}}=\left.y^{\prime}\right|_{T_{0}},\left.b^{\prime \prime}\right|_{T_{0}}=\left.y^{\prime \prime}\right|_{T_{0}}$ and $\left.b^{\prime}\right|_{R_{0}}=\left.b^{\prime \prime}\right|_{R_{0}}$, we have $f\left(x, y^{\prime}\right)=f\left(x, b^{\prime}\right)$, $f\left(x, b^{\prime \prime}\right)=f\left(x, y^{\prime \prime}\right)$ and $f\left(x, b^{\prime}\right)=f\left(x, b^{\prime \prime}\right)$. Hence $f\left(x, y^{\prime}\right)=f\left(x, y^{\prime \prime}\right)$. Thus $f$ is 
concentrated on $R_{0}$ and therefore $f$ depends upon $\kappa$ coordinates in the second variable.

\section{Main results}

Theorem 3.1 Let $X$ be a topological space and let $\kappa$ be an infinite cardinal number. Then the following conditions are equivalent:

(i) every point-finite family of nonempty functionally open in $X$ sets has the cardinality at most $\kappa$;

(ii) $w(Y) \leq \kappa$ for any Valdivia compact space $Y \subseteq C_{p}(X)$;

(iii) $w(Y) \leq \kappa$ for any Eberlein compact space $Y \subseteq C_{p}(X)$.

Proof. $(i) \Longrightarrow(i i)$. By the definition, a Valdivia compact space $Y \subseteq C_{p}(X)$ is homeomorphic to a compact space $Z \subseteq \mathbb{R}^{T}$ such that the set $\{z \in Z$ : $\left.|\operatorname{supp} z| \leq \aleph_{0}\right\}$ is dense in $Z$. Fix a homeomorphism $\varphi: Z \rightarrow Y$. For every $x \in$ $X$ and $z \in Z$ we put $f(x, z)=\varphi(z)(x)$. Clearly, $f$ is a separately continuous function on $X \times Z$. By Theorem 2.1, $f$ depends upon $\kappa$ coordinates in the second variable. This implies that the map $\psi: Z \rightarrow \mathbb{R}^{S}, \psi(z)=\left.z\right|_{S}$, is an injection and hence homeomorphism onto $\tilde{Z}=\psi(Z)$. Therefore $w(Z)=$ $w(\tilde{Z}) \leq \kappa$.

Since every Eberlein compact space is a Valdivia compact space, the implication $($ ii $) \Longrightarrow($ iii $)$ is obvious.

The implication $($ iii $) \Longrightarrow(i)$ was proved in [5, Theorem 1].

Corollary 3.2 Let $X$ be a completely regular space. Then the following conditions are equivalent:

(i) $p(X)=\aleph_{0}$;

(ii) each Valdivia compact space $Y \subseteq C_{p}(X)$ is metrizable.

A topological space $X$ is called pseudocompact if every continuous function $f: X \rightarrow \mathbb{R}$ is bounded; countably compact if a finite subcover can be chosen from any countable open cover of $X$. A topological space $X$ is called $\sigma$-pseudocompact if it is the union of a sequence of pseudocompact subspaces of $X$.

Corollary 3.3 Let $X$ be a completely regular space with $p(X)=\aleph_{0}$ which consists a $\sigma$-pseudocompact dense subspace. Then every countably compact 
space $Y \subseteq C_{p}(X)$ is metrizable.

Proof. By [10, Theorem III.4.23] every countably compact space $Y \subseteq C_{p}(X)$ is an Eberlein compact space. It remains to use Corollary 3.2.

By $\mathcal{K}(X)$ we denote the system of all compact subsets of a topological space $X$. A topological space $X$ is called $\mathcal{K}$-countably-determined if there exist a subset $Y$ in the topological space $\mathbb{N}^{\mathbb{N}}$ and a set-valued mapping $F: Y \rightarrow \mathcal{K}(X)$ such that for every open in $X$ set $U$ the set $\{y \in Y: F(y) \subseteq U\}$ is open in $Y$ and $X=\bigcup_{y \in Y} F(y)$.

Corollary 3.4 Let $X$ be a completely regular space with $p(X)=\aleph_{0}$ which contains a $\mathcal{K}$-countably-determined dense subspace. Then every compact space $Y \subseteq C_{p}(X)$ is metrizable.

Proof. Fix a dense in $X \mathcal{K}$-countably-determined subspace $\tilde{X}$ and a compact space $Y \subseteq C_{p}(X)$. Consider the continuous identity mapping $\varphi: C_{p}(X) \rightarrow$ $C_{p}(\tilde{X})$. Put $\tilde{Y}=\varphi(Y)$. It follows from density $\tilde{X}$ in $X$ that $\left.\varphi\right|_{Y}$ is a bijection and by [11, Theorem 3.1.13] $\varphi$ is a homeomorphism. By [12, Theorem 3.7] $\tilde{Y}$ is a Corson compact space. Thus $Y$ is a Corson compact space and by Corollary 3.2 it is metrizable.

Let $D$ be a discrete space of cardinality $\kappa>\aleph_{0}$. By $L_{\kappa}$ we will denote an one-point Lindelöffication of $D$, that is $L_{\kappa}=D \bigcup\{\infty\}$ and all neighborhoods of $\infty$ in $L_{\kappa}$ are the complements in $L_{\kappa}$ to all at most countable subsets of $D$, besides the discrete topology on $D$ coincides with the topology on $D$ induced by $L_{\kappa}$. A topological space $X$ is called primary Lindelöff if $X$ is a continuous image of a closed subset of the topological product $L_{\kappa}{ }^{\mathbb{N}}$ for some $\kappa>\aleph_{0}$. Note that by [10, Corollary IV.3.17] for a primary Lindelöff space $X$ every compact space $Y \subseteq C_{p}(X)$ is a Corson compact space.

The following result can be obtained analogously.

Corollary 3.5 Let $X$ be a completely regular space with $p(X)=\aleph_{0}$ which contains a primary Lindelöff dense subspace. Then every compact space $Y \subseteq$ $C_{p}(X)$ is metrizable.

Recall that a set $F$ of functions defined on a set $X$ separates points on $X$ if for arbitrary distinct points $x^{\prime}, x^{\prime \prime} \in X$ there exists a function $f \in F$ such that $f\left(x^{\prime}\right) \neq f\left(x^{\prime \prime}\right)$.

Theorem 3.6 Let $Y$ be a Valdivia compact space and let $\kappa$ be an infinite cardinal number. Then the following conditions are equivalent:

(i) $w(Y) \leq \kappa ;$ 
(ii) $p\left(C_{p}(Y)\right) \leq \kappa$;

(iii) there exists a space $X \subseteq C_{p}(Y)$ with $p(X) \leq \kappa$ which separates points on $Y$.

Proof. $(i) \Longrightarrow(i i)$. Suppose that $w(Y) \leq \kappa$. If $Y$ is infinite then $d\left(C_{p}(Y)\right) \leq$ $w(Y) \leq \kappa$. If $Y$ is finite then $d\left(C_{p}(Y)\right)=\aleph_{0} \leq \kappa$ anyway. Thus $d\left(C_{p}(Y)\right) \leq \kappa$. Therefore $p\left(C_{p}(Y)\right) \leq \kappa$.

The implication $(i i) \Longrightarrow($ iii $)$ is obvious.

$($ iii $) \Longrightarrow(i)$. Fix a subspace $X \subseteq C_{p}(Y)$ with $p(X) \leq \kappa$ which separates points on $Y$. Consider a continuous mapping $\varphi: Y \rightarrow C_{p}(X), \varphi(y)(x)=x(y)$. Since $X$ separates points on $Y$, the map $\varphi: Y \rightarrow \varphi(Y)$ is a bijection and by [11, Theorem 3.1.13] $\varphi$ is a homeomorphism. Thus $\varphi(Y)$ is an Valdivia compact space and $w(Y) \leq \kappa$ by Theorem 3.1 .

Note that Theorem 3.6 is not true for an arbitrary compact space $Y$. Indeed, $2^{\aleph_{0}}=w(Y)>p\left(C_{p}(Y)\right)=\aleph_{0}$ for $Y=\beta \mathbb{N}$ (see [6], proof of Theorem 2.11).

Corollary 3.7 Any Valdivia compact space $Y$ is metrizable if and only if $p\left(C_{p}(Y)\right)=\aleph_{0}$.

\section{Linearly ordered compacta}

Let $(X,<)$ be a linearly ordered space. For every $x, y \in X, x<y$, we put $(x, y)=\{z \in X: x<z<y\},[x, y)=\{z \in X: x \leq z<y\},(x, y]=\{z \in X:$

$x<z \leq y\}$ and $[x, y]=\{z \in X: x \leq z \leq y\}$. Elements $x, y \in X, x<y$ such that $(x, y)=\varnothing$ are called neighbor points.

Proposition 4.1 Let $(X,<)$ be a linearly ordered countably compact space and let $A=\left\{(x, y) \in X^{2}: x<y\right.$ and $\left.(x, y)=\varnothing\right\}$. Then $c(X) \leq p\left(C_{p}(X)\right)$ and $|A| \leq p\left(C_{p}(X)\right)$.

Proof. Let $B=\left\{\left(x_{i}, y_{i}\right) \in X^{2}: i \in I\right\}$ be a set which satisfies the following conditions:

a) $x_{i}<y_{i}$ for every $i \in I$;

b) $y_{i} \leq x_{j}$ or $y_{j} \leq x_{i}$ for every distinct $i, j \in I$.

Put $U_{i}=\left\{f \in C_{p}(X): f\left(x_{i}\right)<0\right.$ and $\left.f\left(y_{i}\right)>1\right\}$ for each $i \in I$. We show that $\left(U_{i}: i \in I\right)$ is a point-finite family in $C_{p}(X)$. Suppose that $f \in C_{p}(X)$ such that a set $J=\left\{i \in I: f \in U_{i}\right\}$ is infinite. Then in an infinite set $\left\{x_{i}: i \in J\right\}$ 
there exists a strictly increasing or decreasing sequence $\left(x_{i_{n}}\right)_{n=1}^{\infty}, i_{n} \in J$. Since $X$ is a countably compact space, there exists $z \in X$ such that $z=\lim _{n \rightarrow \infty} x_{i_{n}}=$ $\lim _{n \rightarrow \infty} y_{i_{n}}$. Therefore $f(z)=\lim _{n \rightarrow \infty} f\left(x_{i_{n}}\right) \leq 0$ and $f(z)=\lim _{n \rightarrow \infty} f\left(y_{i_{n}}\right) \geq 1$, which is impossible.

Thus $\left(U_{i}: i \in I\right)$ is a point-finite family and $|I| \leq p\left(C_{p}(X)\right)$. Since any chain generates a set $B$ with $\left(x_{i}, y_{i}\right) \neq \varnothing$ for every $i \in I$, and $A$ generates a set $B$ with $\left(x_{i}, y_{i}\right)=\varnothing$ for every $i \in I$, we have the inequality $c(X) \leq p\left(C_{p}(X)\right)$ and $|A| \leq p\left(C_{p}(X)\right)$.

The following assertion seems to be known.

Proposition 4.2 Let $(X,<)$ be a linearly ordered space. Then $d(X) \leq c\left(X^{2}\right)$.

Proof. Let $A$ be a set of all isolated points of $X$ and let $\left(W_{i}: i \in I\right)$ be a maximal family of nonempty sets $W_{i}=\left(a_{i}, b_{i}\right) \times\left(c_{i}, d_{i}\right)$ in $X^{2}$ such that $b_{i} \leq c_{i}$ or $d_{i} \leq a_{i}$ for each $i \in I$. Clearly $|A| \leq c\left(X^{2}\right)$ and $|I| \leq c\left(X^{2}\right)$. Put $B=A \cup\left\{a_{i}, b_{i}, c_{i}, d_{i}: i \in I\right\}$. Suppose that $U=X \backslash \bar{B} \neq \varnothing$.

Choose a nonempty set $(x, y) \subseteq U$. Since $U \cap A=\varnothing$ then there exist $a, b, c, d \in$ $(x, y)$ such that $a<b \leq c<d$ and sets $(a, b)$ and $(c, d)$ are nonempty. Then an open in $X^{2}$ nonempty set $W=(a, b) \times(c, d)$ such that $W \cap W_{i}=\varnothing$ for every $i \in I$. This contradicts the maximality of $\left(W_{i}: i \in I\right)$.

Theorem 4.3 Let $(X,<)$ be an infinite linearly ordered compact. Then $w(X)=$ $p\left(C_{p}(X)\right)$.

Proof. The inequality $p\left(C_{p}(X)\right) \leq w(X)$ can be proved like in the proof of Theorem 3.6.

Let $p\left(C_{p}(X)\right)=\kappa$. Then a set $\tilde{A}=\left\{(x, y) \in X^{2}: x<y\right.$ and $\left.(x, y)=\varnothing\right\}$ has the cardinality at most $\kappa$ by Proposition 4.1. Therefore a set $A=\{x, y \in X$ : $(x, y) \in \tilde{A}\}$ has the cardinality at most $\kappa$. Note that for arbitrary dense in $X$ set $B$ the system $\{(x, y): x<y, x, y \in A \cup B\} \bigcup\{[a, x): x \in A \cup B\} \bigcup\{(x, b]$ : $x \in A \cup B\}$, where $a=\min X$ and $b=\max X$, is a base of open in $X$ sets. Therefore it is sufficient to prove that $d(X) \leq \kappa$.

Consider a system $\mathcal{V}$ of all open in $X$ nonempty set $V$ with $d(V) \leq \kappa$. Choose a maximal disjoint system $\mathcal{U} \subseteq \mathcal{V}$. Proposition 4.1 implies $|\mathcal{U}| \leq \kappa$. For every $U \in \mathcal{U}$ we choose a set $B_{U} \subseteq U$ such that $\left|B_{U}\right| \leq \kappa$ and $U \subseteq \overline{B_{U}}$. Put $B=A \cup \bigcup_{U \in \mathcal{U}} B_{U}$ and $Y=\bar{B}$. Then $|B| \leq \kappa^{2}=\kappa$.

Suppose that $X \backslash Y \neq \varnothing$. Choose $x_{0}, y_{0} \in X \backslash Y$ such that $x_{0}<y_{0}$ and $X_{0}=\left[x_{0}, y_{0}\right] \subseteq X \backslash Y$. We show that $c\left(X_{0}^{2}\right) \leq \kappa$. 
Let $\left(W_{i}: i \in I\right)$ be a disjoint family of open sets in $X_{0}^{2}$. Suppose that $|I|>\kappa$. For every $i \in I$ choose pair-wise distinct points $a_{i}, b_{i}, c_{i}, d_{i} \in X_{0}$ such that $a_{i}<$ $b_{i}, c_{i}<d_{i}$ and $\left(a_{i}, b_{i}\right) \times\left(c_{i}, d_{i}\right) \subseteq W_{i}$ (it is possible since $X_{0}$ has no neighbor point). Put $U_{i}=\left\{f \in C_{p}\left(X_{0}\right): f\left(a_{i}\right)<0, f\left(b_{i}\right)>1, f\left(c_{i}\right)<0, f\left(d_{i}\right)>1\right\}$ for every $i \in I$. Note that $p\left(C_{p}\left(X_{0}\right)\right) \leq \kappa$, thus $\left(U_{i}: i \in I\right)$ is not point-finite in $C_{p}\left(X_{0}\right)$. There exist a function $f_{0} \in C_{p}\left(X_{0}\right)$ and an infinite set $J \subseteq I$ such that $f_{0} \in U_{i}$ for every $i \in J$. Since $f_{0}$ is continuous on linearly ordered compact space $X_{0}$ which has no neighbor point, there exists a finite set $K \subseteq X_{0}$ such that for every $x, y \in X_{0}$ the inequality $\left|f_{0}(x)-f_{0}(y)\right|>1$ implies $(x, y) \cap K \neq$ $\emptyset$. Then $\left(a_{i}, b_{i}\right) \cap K \neq \varnothing,\left(c_{i}, d_{i}\right) \cap K \neq \varnothing$ therefore $W_{i} \cap K^{2} \neq \varnothing$ for every $i \in J$ which contradicts the disjointness of $\left(W_{i}: i \in I\right)$. Hence $|I| \leq \kappa$.

Thus $c\left(X_{0}^{2}\right) \leq \kappa$ and according to Proposition 4.2 we obtain that $d\left(X_{0}\right) \leq \kappa$, which contradicts the maximality of the system $\mathcal{U}$. Therefore $X \backslash Y=\varnothing$, $d(X) \leq \kappa$ and $w(X) \leq \kappa$.

The following corollary gives a negative answer to Question 5.2 from [7].

Corollary 4.4 A linearly ordered compact $X$ is metrizable if and only if $p\left(C_{p}(X)\right)=\aleph_{0}$.

\section{References}

[1] W. Moran Separate continuity and support of measures, J. London. Math. Soc. 44 (1969), 320-324.

[2] G. Vera Baire measurability of separately continuous functions, Quart. J. Math. Oxford. 39, 153 (1988), 109-116.

[3] V.V. Mykhaylyuk, O.V. Sobchuk Baire classification of separately continuous functions and dependence upon countable number of coordinates, Nauk. Visn. Chern. Univ. Vyp. 191-192, Matem., Chernivtsi: Ruta (2004), 116-118 (in Ukrainian).

[4] V.V. Tkachuk Cardinal invariants of the Suslin number type, Dokl. Akad. Nauk SSSR. 270, 4 (1983), 795-798 (in Russian).

[5] V.V. Tkachuk A supertopological cardinal invariants, Vestnik Moskov. Univ. Ser. I Mat. Mekh. 4 (1984), 26-29(in Russian).

[6] A.V. Arhangel'skii, V.V. Tkachuk V.V. Calibers and point-finite cellularity of the space $C_{p}(X)$ and some questions of S.Gul'ko and M.Husek, Topology Appl. 23, 1 (1986), 65-73.

[7] O.G. Okunev, V.V. Tkachuk Lindelöf $\Sigma$-property in $C_{p}(X)$ and $p\left(C_{p}(X)\right)=\omega$ do not imply countable network weight in $X$, Acta Math. Hungar. 90, 1-2 (2001), 119-132. 
[8] N.D. Kalamidas, G.D. Spiliopoulos Compact sets in $C_{p}(X)$ and calibers, Can. Math. Bull. 35, 4 (1992), 497-502.

[9] V.V. Mykhaylyuk One-point discontinuity set of separately continuous functions on the product of two compact spaces, Ukr. Mat. Zhurn. 57, 1 (2005), 94-101 (in Ukrainian).

[10] A.V. Arhangelskii Topological spaces of functions, .: Izd-vo Moskovskogo Univ. (1989), 222 s.(in Russian).

[11] R. Engelking General topology, M.: Mir (1986), 752 s. (in Russian).

[12] M. Talagrand Espaces de Banach faiblement $\mathcal{K}$-analytiques, Ann. of Math. 110 (1979), 407-438. 